University of Wollongong

Research Online

Australian Institute for Innovative Materials -

Papers

Australian Institute for Innovative Materials

$1-1-2018$

Control of superconductivity by means of electric-field-induced strain in superconductor/piezoelectric hybrids

D Stamopoulos

Demokritos National Centre For Scientific Research

M Zeibekis

Demokritos National Centre For Scientific Research

Shujun Zhang

University of Wollongong, shujun@uow.edu.au

Follow this and additional works at: https://ro.uow.edu.au/aiimpapers

Part of the Engineering Commons, and the Physical Sciences and Mathematics Commons

Research Online is the open access institutional repository for the University of Wollongong. For further information contact the UOW Library: research-pubs@uow.edu.au 


\title{
Control of superconductivity by means of electric-field-induced strain in superconductor/piezoelectric hybrids
}

\author{
Abstract \\ The controlled modification of superconductivity by any means, specifically in hybrid systems, has \\ attracted much interest in the recent decades. Here, we present experimental data and phenomenological \\ modeling on the control of $\mathrm{T} \mathrm{C}$ of superconducting (SC) Nb thin films, with thickness $3 \mathrm{~nm} \leq \mathrm{dNb} \leq 50 \mathrm{~nm}$, \\ under the application of in-plane strain, $S(E$ ex $)$ induced by an external out-of-plane electric field, $E$ ex to \\ piezoelectric (PE) single crystals, namely, (1-x) Pb(Mg 1/3 Nb 2/3 ) 3 -xPbTiO 3 (PMN-xPT), with $x=0.27$ \\ and 0.31 . We report experimental modification of $\mathrm{T} \mathrm{C}$ of $\mathrm{Nb}$ by $\mathrm{E}$ ex, accurately described by a \\ phenomenological model that incorporates the constitutive relation S(E ex ) of PMN-xPT. The systematic \\ experimental-phenomenological modeling approach introduced here is generic and paves the way for an \\ understanding of the underlying physical mechanisms in any SC/PE hybrid.

\section{Disciplines} \\ Engineering | Physical Sciences and Mathematics

\section{Publication Details} \\ Stamopoulos, D., Zeibekis, M. \& Zhang, S. J. (2018). Control of superconductivity by means of electric- \\ field-induced strain in superconductor/piezoelectric hybrids. Journal of Applied Physics, 123 (2), \\ 023903-1-023903-8.
}




\section{Control of superconductivity by means of electric-field-induced strain in superconductor/piezoelectric hybrids}

D. Stamopoulos, M. Zeibekis, and S. J. Zhang

Citation: Journal of Applied Physics 123, 023903 (2018);

View online: https://doi.org/10.1063/1.5005045

View Table of Contents: http://aip.scitation.org/toc/jap/123/2

Published by the American Institute of Physics

\section{Articles you may be interested in}

Multiphase magnetic systems: Measurement and simulation

Journal of Applied Physics 123, 023902 (2018); 10.1063/1.5010799

Photonic nanojet assisted enhancement of Raman signal: Effect of refractive index contrast

Journal of Applied Physics 123, 023102 (2018); 10.1063/1.4994944

Optical nutation in acetylene-filled hollow-core photonic crystal fiber

Journal of Applied Physics 123, 023101 (2018); 10.1063/1.5004179

Modeling of reduced secondary electron emission yield from a foam or fuzz surface

Journal of Applied Physics 123, 023302 (2018); 10.1063/1.5008261

Unravelling radiative energy transfer in solid-state lighting

Journal of Applied Physics 123, 023103 (2018); 10.1063/1.5008922

Probing dynamic behavior of electric fields and band diagrams in complex semiconductor heterostructures Journal of Applied Physics 123, 024301 (2018); 10.1063/1.5013274

\section{Scilight}

Sharp, quick summaries illuminating the latest physics research

\section{Sign up for FREE!}

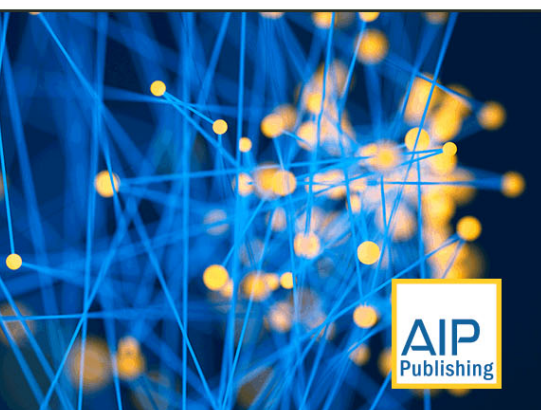




\title{
Control of superconductivity by means of electric-field-induced strain in superconductor/piezoelectric hybrids
}

\author{
D. Stamopoulos, ${ }^{1,2, a)}$ M. Zeibekis, ${ }^{2}$ and S. J. Zhang ${ }^{3}$ \\ ${ }^{1}$ Department of Solid State Physics, National and Kapodistrian University of Athens, \\ Zografou Panepistimioupolis, 15784 Zografou, Greece \\ ${ }^{2}$ Institute of Nanoscience and Nanotechnology, National Center for Scientific Research 'Demokritos', \\ 15310 Aghia Paraskevi, Greece \\ ${ }^{3}$ Institute for Superconducting and Electronic Materials, Australian Institute of Innovative Materials, \\ University of Wollongong, NSW 2500, Australia
}

(Received 16 September 2017; accepted 26 December 2017; published online 11 January 2018)

The controlled modification of superconductivity by any means, specifically in hybrid systems, has attracted much interest in the recent decades. Here, we present experimental data and phenomenological modeling on the control of $\mathrm{T}_{C}$ of superconducting ( $\left.\mathrm{SC}\right) \mathrm{Nb}$ thin films, with thickness $3 \mathrm{~nm} \leq \mathrm{d}_{N b} \leq 50 \mathrm{~nm}$, under the application of in-plane strain, $S\left(\mathrm{E}_{e x}\right)$ induced by an external out-of-plane electric field, $\mathrm{E}_{e x}$ to piezoelectric $(\mathrm{PE})$ single crystals, namely, $(1-x) \mathrm{Pb}\left(\mathrm{Mg}_{1 / 3} \mathrm{Nb}_{2 / 3}\right) \mathrm{O}_{3^{-}}$ $\mathrm{xPbTiO}_{3}(\mathrm{PMN}-\mathrm{xPT})$, with $\mathrm{x}=0.27$ and 0.31 . We report experimental modification of $\mathrm{T}_{C}$ of $\mathrm{Nb}$ by $\mathrm{E}_{e x}$, accurately described by a phenomenological model that incorporates the constitutive relation $S\left(\mathrm{E}_{e x}\right)$ of PMN-xPT. The systematic experimental-phenomenological modeling approach introduced here is generic and paves the way for an understanding of the underlying physical mechanisms in any SC/PE hybrid. Published by AIP Publishing. https://doi.org/10.1063/1.5005045

\section{INTRODUCTION}

The control of superconductivity, by any means, is a long-standing pursuit since the early days of its discovery. In this context, ferromagnetism, ${ }^{1-4}$ pressure, ${ }^{5-7}$ and strain (static, induced by the substrate or grain boundaries) ${ }^{8-10}$ have been recruited, among others, as candidate control parameters. However, pressure and static strain cannot be employed to serve applications relying on the non-volatile control of superconducting (SC) devices scalable at the nano-range. ${ }^{11-13}$ Recently, hybrid structures consisting of ferromagnetic films adjacent to piezoelectric (PE) substrates have been investigated on how the magnetic properties of the former can be controlled by the strain imposed by the latter. ${ }^{14-19}$ The relevant hybrids of PE and superconducting (SC) constituents have not been explored, in detail, so far (see Refs. 20-23 for some relevant work). In such hybrids (e.g., SC/PE/SC), it is expected that the strain induced in the $\mathrm{PE}$ interlayer by the electric field, easily applied by a control voltage, will penetrate to the adjacent SC outer layers, thus distorting their crystal lattice. In this way, in conventional low- $\mathrm{T}_{C}$ SC one could modify the phonon branches, thus affecting $\mathrm{T}_{C} \cdot{ }^{24}$ Specifically, the characteristics of phonon branches depend on two parameters, namely, the mass of atoms and the lattice constants. Since the former cannot be altered in real time, it is only the latter that can be modified, e.g., in SC/PE/SC hybrids, to control superconductivity.

According to Refs. 25-27 the $\mathrm{T}_{C}$ of a conventional SC, in the strong coupling regime, is given by the relation

$$
k_{B} T_{C}=\frac{\hbar \omega_{l n}}{1.20} \exp \left(-\frac{1.04(1+\lambda)}{\lambda-\mu^{*}(1+0.62 \lambda)}\right),
$$

\footnotetext{
${ }^{\text {a) }}$ Author to whom correspondence should be addressed: densta@phys.uoa.gr and d.stamopoulos@inn.demokritos.gr
}

where $\mathrm{k}_{B}$ is the Boltzmann constant and $\hbar$ is the Planck constant (in what follows, we have set $\mathrm{k}_{B}=\hbar=1$ ), $\omega_{l n}$ is a mean frequency of phonons, and the parameters $\lambda$ and $\mu^{*}$ refer to the attractive (phonon-mediated) and repulsive (Coulomb) interactions, respectively, between Cooper-paired electrons. ${ }^{24-27}$

Based on relation (1), it is plausible to suggest that one could modify the critical temperature, $\mathrm{T}_{C}$ of a SC by applying controlled strain, represented by the "Strain-Electric field" constitutive relation $S\left(\mathrm{E}_{e x}\right)$, that develops to an adjacent PE substrate upon application of an external electric field, $\mathrm{E}_{e x}$. Accordingly, in the present work, we studied SC/ $\mathrm{PE} / \mathrm{SC}$ hybrids by using $\mathrm{SC}$ thin films of $\mathrm{Nb}$ and $\mathrm{PE}$ single crystals of $(1-x) \mathrm{Pb}\left(\mathrm{Mg}_{1 / 3} \mathrm{Nb}_{2 / 3}\right) \mathrm{O}_{3}-\mathrm{xPbTiO}_{3}$ (PMN-xPT), produced as discussed in Refs. 4 and 28, respectively. Specifically, we employed PMN-xPT single crystals of different PT content, with $\mathrm{x}=0.27$ and 0.31 , to investigate the impact of electro-mechanical stimuli of different magnitude on superconductivity; piezoelectric activity is maximum near the so-called morphotropic phase boundary of optimum PT content (i.e., $\mathrm{x}=0.31$ ), while reduces drastically away from it (i.e., $x=0.27$ ). ${ }^{28}$ Also, we focused on thin films of $\mathrm{Nb}$, with thickness $3 \mathrm{~nm} \leq \mathrm{d}_{N b} \leq 50 \mathrm{~nm}$, since it is expected that only in this case the SC will experience throughout its whole thickness, the structural distortion imposed by the adjacent PMN-xPT single crystal. To estimate the overall volume change induced to the $\mathrm{Nb}$ thin films we have to take into account both the change over the ab-plane (surface change) and of the change along the c-axis. Obviously, the change of the $\mathrm{Nb}$ films surface must follow the change of the PMN-PT crystal surface. On the other hand, although in general is risky to discuss the change along the c-axis of $\mathrm{Nb}$ films, in our case, it is reasonable to assume that it also follows the respective change along the c-axis of the PMN-PT 
crystal for two reasons: first, the thickness of the Nb films is very small so that the strain perturbation experienced at the $\mathrm{PMN}-\mathrm{PT} / \mathrm{Nb}$ inner interface spans the entire thickness of the $\mathrm{Nb}$ thin films until the $\mathrm{Nb} /$ vacuum outer surface. Second, in the particular set of PMN-xPT crystals, the application of an external electric field induces a major strain over the abplane and only a minor strain along the c-axis.

The experimental data demonstrated modification of $\mathrm{T}_{C}$ by $\mathrm{E}_{e x}$ that followed the magnitude of $\mathrm{S}\left(\mathrm{E}_{e x}\right)$ of the employed PMN-xPT single crystals of different PT content $(x=0.27$ and 0.31). Theoretically, we phenomenologically modeled the experimental data of $\mathrm{T}_{C}$ on $\mathrm{E}_{e x}$ through appropriately chosen trial functions for $\mathrm{T}_{C}(\mathrm{~S})$. The latter, upon incorporation of the experimentally determined $S\left(\mathrm{E}_{e x}\right)$, evolves to a closed-form relation for $\mathrm{T}_{C}\left(\mathrm{E}_{e x}\right)$ that reproduces excellently the experimental data by using strictly reasonable fitting parameters.

\section{EXPERIMENTAL DETAILS}

\section{A. PMN-xPT single crystals}

The PMN-xPT single crystals, with $\mathrm{x}=0.27$ and 0.31 , $\approx 100 \mathrm{~mm}$ in diameter and $>150 \mathrm{~mm}$ in length, were grown along the [010] crystallographic direction by the seeded Bridgman technique. Samples with a rectangular shape were oriented along the [100] and [011] directions by real-time Laue X-ray, following the IEEE standard, where the large face is [011] $(\mathrm{z}(3))$, while the length direction is [100] $(\mathrm{y}(2))$ and the width direction is [011] $(\mathrm{x}(1))$. Both the PMN-xPT $(x=0.27$ and 0.31$)$ are in the rhombohedral phase, with the latter one being closer to the rhombohedral to orthorhombic morphotropic phase boundary. With applying electric field on the (011) face, the crystals are in an engineered domain configuration, with the macroscopic symmetry of $\mathrm{mm} 2$, where the in-plane displacement $\left(\mathrm{d}_{32}\right)$ is negative that is compressive along the $\mathrm{y}(2)$ direction, while the in-plane displacement $\left(d_{31}\right)$ is positive that is expansive along the $x(1)$ direction. It should be noted that the out-of-plane displacement $\left(d_{33}\right)$ is also positive that is expansive along $\mathrm{z}(3)$, but smaller than the $d_{32}$. More details can be found in Ref. 28 .
After preparation, the single crystals were left unpoled and were finally cut to the proper dimensions $(x, y, z)=(5 \times 6$ $\times 0.4-0.5 \mathrm{~mm}^{3}$ ) [see the coordinate system in Fig. 1(a)]. To reduce surface roughness, the crystals were subjected to mild polishing, wherever appropriate.

\section{B. Nb thin films}

The $\mathrm{Nb}$ thin films, with thickness in the range $3 \mathrm{~nm}$ $\leq \mathrm{d}_{N b} \leq 50 \mathrm{~nm}$, were prepared on PMN-xPT single crystals and on reference $\mathrm{Si}$ [100] substrates by using a high-purity $\mathrm{Nb}$ target (Materion, $\mathrm{OH}, \mathrm{USA}$ ) with a magnetron dc sputtering unit Edwards 306A (Edwards, Sanborn, NY, USA). Details can be found in Ref. 29. Special attention was paid to prepare high-quality $\mathrm{Nb}$ thin films that preserve superconductivity for the lowest thickness possible. In this respect, a specially designed deposition protocol was employed (see Sec. I of the supplementary material).

\section{Estimation of the "Strain-Electric field" curve}

The "Strain-Electric field" curve, $\mathrm{S}\left(\mathrm{E}_{e x}\right)$ that describes the electro-mechanical response of the PE PMN-xPT single crystals was obtained with standard global and local techniques. $^{28,30,31}$ Referring to the global measurements, the small signal piezoelectric coefficients were measured using an HP4194A Impedance Analyzer (Palo Alto, CA, USA) and calculated according to the IEEE standard on piezoelectricity. The large signal piezoelectric coefficient was evaluated by the slope of the strain-electric field curve, while the displacement was measured by a homemade Linear Variable Differential Transducer. Referring to the local technique, it was originally introduced in Ref. 30 for PMN-PT single crystals and further elaborated in Ref. 31 for $\mathrm{Pb}\left(\mathrm{Zr}_{0.52} \mathrm{Ti}_{0.48}\right) \mathrm{O}_{3}$ polycrystalline samples. This method is based on the comparison of consecutive images recorded either with Optical Microscopy (OM) or Atomic Force Microscopy (AFM) upon variation of the external electric field, $\mathrm{E}_{e x}$ (an example is presented in Sec. II of the supplementary material). Specifically, the method lies in the direct comparison of the images
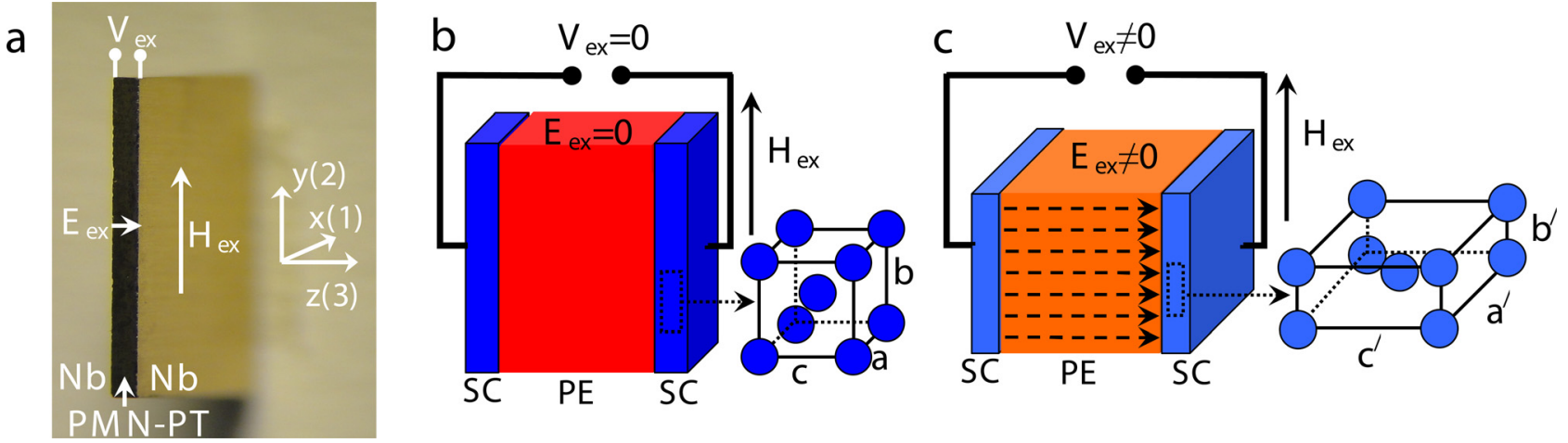

FIG. 1. (a) Photographic illustration of an Nb/PMN-xPT/Nb hybrid subjected to an external voltage, $\mathrm{V}_{e x}$. A schematic coordinate system is shown to specify, first, the directions where the external electric, $\mathrm{E}_{e x}$ and magnetic, $\mathrm{H}_{e x}$ fields are applied (out-of-plane and in-plane, respectively) and, second, the electromechanical response of the PMN-xPT single crystals upon application of $\mathrm{E}_{e x}$. (b) and (c) Schematic illustration of a SC/PE/SC hybrid for b zero and c nonzero external electric field, $\mathrm{E}_{e x}$. The two SC outer layers, thickness-overtoned for the sake of presentation, serve as electrodes for the application of the external voltage, $\mathrm{V}_{e x}$ that generates an $\mathrm{E}_{e x}$ normal to the surface of the $\mathrm{SC} / \mathrm{PE} / \mathrm{SC}$ hybrid (parallel to the $\mathrm{Z}$-axis, that is out-of-plane). The external magnetic field, $\mathrm{H}_{e x}$ is applied parallel to the surface of the SC/PE/SC hybrid (parallel to the xy-plane that is in-plane). The in-plane electro-mechanical response of the PE interlayer (expansive along $\mathrm{x}$ and $\mathrm{z}$ axes and compressive along the $\mathrm{y}$ axis) is imposed to the lattice constants of the SC outer layers, modifying them from a, $\mathrm{b}$, and $\mathrm{c}$ for $\mathrm{E}_{e x}=0$ to $\mathrm{a}^{\prime}>\mathrm{a}, \mathrm{b}^{\prime}<\mathrm{b}$, and $\mathrm{c}^{\prime}>\mathrm{c}$ for $\mathrm{E}_{e x} \neq 0$. 
recorded prior to and after the variation of $\mathrm{E}_{e x}$ that enables us to directly measure the developed deformations at the micrometer (with OM) and nanometer (with AFM) level. From these measurements, we can estimate the $\mathrm{d}_{i j}$ coefficients in a direct way by using simple algebraic calculations. ${ }^{30,31}$ In this way, the precise in-plane compressive $\mathrm{S}_{2}\left(\mathrm{E}_{e x, 3}\right)$ curves were obtained for the specific PMN-xPT single crystals employed here, so that a reliable comparison can be directly performed with the $\mathrm{T}_{C}\left(\mathrm{E}_{e x}\right)$ data obtained for the $\mathrm{Nb}$ thin films through magnetization measurements.

As mentioned above, the rectangular shaped PMN-xPT crystals have been cut along specific crystallographic planes. The crystallographic orientation of PMN-xPT crystals promotes the development of a preferable global piezoelectric strain along a specific direction, that in our case, lies along the ab-plane. This preferable global behavior of strain in PMNXPT does not describe accurately the response of strain at the local level, where different local areas exhibit nonhomogeneous development of strain as was evidenced in a recent publication of ours. ${ }^{30}$ Therefore, it is reasonable to assume that this local non-homogeneous development of strain in PMN-xPT is also transferred to the adjacent $\mathrm{Nb}$ thin films, leading to a non-homogeneous variation of their $\mathrm{T}_{C}$, at the local level. In addition, due to the relatively high surface roughness of the PMN-xPT crystals (especially in their non-polished state), the deposited $\mathrm{Nb}$ thin films should be highly polycrystalline a fact that further promotes the non-homogeneous variation of the recorded $\mathrm{T}_{C}$. However, the performed magnetization measurements by means of SQUID (see below) cannot detect the influence of the non-homogeneous strain on $\mathrm{T}_{C}$ of the $\mathrm{Nb}$ thin films; the SQUID magnetometer is a global technique that measures the mean value of the magnetic moment originating from the entire sample. This means that the modification of critical temperature, $\mathrm{T}_{C}$, from the strain, $\mathrm{S}\left(\mathrm{E}_{e x}\right)$, originating from the application of an external electric field, $\mathrm{E}_{e x}$, to the $\mathrm{Nb}$ / $\mathrm{PMN}-\mathrm{xPT} / \mathrm{Nb}$ hybrids studied in this work, actually refers to the mean value of "global, $\mathrm{T}_{C}$ " and to the respective mean value of "global strain, $\mathrm{S}\left(\mathrm{E}_{e x}\right)$ " (except for the cases where the "local strain" is clearly discussed).

\section{Atomic force microscopy}

AFM was employed to estimate the $\mathrm{S}\left(\mathrm{E}_{e x}\right)$ response of the PE PMN-xPT single crystals at the local level (see above, Sec. II of the supplementary material, and Refs. 30 and 31). AFM data were acquired on non-polished and polished PMN-xPT single crystals at room temperature $\mathrm{T}=300 \mathrm{~K}$ with a scanning probe microscope Solver PRO (NT-MDT Co, Moscow, Russia) in semi-contact mode by using cantilevers NCHR (Nano and More GmbH, Wetzlar, Germany). The mean surface roughness, Sa, was calculated by using the instrument software. Details can be found in Refs. 30 and 31 .

\section{E. Optical microscopy}

OM was also employed to estimate the $\mathrm{S}\left(\mathrm{E}_{e x}\right)$ response of the PE PMN-xPT single crystals at the local level (see above and Refs. 30 and 31) The OM data were obtained by means of a LEICA DMRXP (Leica, Wetzlar, Germany) and an ORTHOLUX (Leitz, Wetzlar, Germany) in reflection light operation. The magnification used was within 200-600 (objective lens 20-60).

\section{F. Magnetization measurements}

Magnetization measurements were performed with a SQUID magnetometer MPMS 5.5 Tesla (Quantum Design, San Diego, CA, USA). Both isofield m(T) curves, zero-field cooled (ZFCs), and field cooled curves (FCs), were recorded for a dc external magnetic field, $\mathrm{H}_{e x}$ applied parallel to the surface (in-plane) of the Nb/PMN-xPT/Nb samples. For the application of the dc external voltage, $\mathrm{V}_{e x}$ to the PMN-xPT single crystals, a homemade sample rod was constructed (see Sec. III of the supplementary material). The outer $\mathrm{Nb}$ thin films were used as electrodes. Thus, in this configuration, the dc external electric field, $\mathrm{E}_{e x}$, was normal to the surface (outof-plane) of the Nb/PMN-xPT/Nb samples. For details, see Figs. 1(a)-1(c), below.

A dc leakage current could appear during our magnetization measurements due to even partial dielectric breakdown of the insulator PMN-xPT crystals by the applied dc voltage. Since such a dc leakage current could affect the critical temperature, $\mathrm{T}_{C}$, of the $\mathrm{Nb}$ thin films, ${ }^{32}$ during our experiments, we employed an amperometer of sensitivity $0.1 \mu \mathrm{A}$ to monitor this issue. Previous publications of ours evidenced that superconductivity of $\mathrm{Nb}$ films is affected by a dc current above $1 \mathrm{~mA}$ [for instance, see Fig. 12(b) in Ref. 32] Accordingly, in the present experiments, we ensured that the $\mathrm{Nb}$ thin films were never subjected to an undesired dc electric current higher than 1-5 $\mu \mathrm{A}$, else the measurement was abandoned and the specific $\mathrm{Nb} / \mathrm{PMN}-\mathrm{xPT} / \mathrm{Nb}$ hybrid was rejected.

\section{G. Fitting procedure, errors, and reproducibility}

Here, we clarify the details in the fitting procedures employed in the rest of the paper. Prior to the fitting of an experimental data set of $\mathrm{T}_{C}\left(\mathrm{E}_{e x}\right)$, we fit the detailed experimental data sets $\mathrm{S}\left(\mathrm{E}_{e x}\right)$ coming from the global technique [since $\mathrm{T}_{C}\left(\mathrm{E}_{e x}\right)$ originates from global magnetization measurements, as well], either with a linear [approximation of $\mathrm{S}\left(\mathrm{E}_{e x}\right)$ that is enough to describe the crossover behavior of $\mathrm{T}_{C}\left(\mathrm{E}_{e x}\right)$ ] or quadratic [more accurate $\mathrm{S}\left(\mathrm{E}_{e x}\right)$ that is needed to describe the more complex non-monotonic behavior of the $\left.\mathrm{T}_{C}\left(\mathrm{E}_{e x}\right)\right]$ function. This fixes the multiplying factors that are introduced in the non-primed parameters of $\lambda, \mu$, and $\omega_{l n}$ to transform them to the primed ones. Then, we are using the respective relation of $\mathrm{T}_{C}\left(\mathrm{E}_{e x}\right)$ to fit an experimental data set. Thus, relations $\mathrm{T}_{C}\left(\mathrm{E}_{e x}\right)$ have to describe the experimental points under two restrictions that should be fulfilled simultaneously: by using parameters for $\lambda, \mu$, and $\omega_{l n}$ that are compatible with basic knowledge, while they carry the already imprinted information of the $\mathrm{S}\left(\mathrm{E}_{e x}\right)$ experimental data.

The error bars that accompany each experimental data point of the $\mathrm{T}_{C}\left(\mathrm{E}_{e x}\right)$ curves shown below relate to the uncertainty in the identification of $\mathrm{T}_{C}$ in each isofield $\mathrm{m}(\mathrm{T})$ curve. To define the error bars of each point of $\mathrm{T}_{C}$, we used the specific strict criterion: for each $\mathrm{m}(\mathrm{T})$ curve, the error bar in the determination of $\mathrm{T}_{C}$ was set to be an integer multiple of the employed step in the temperature scan, depending on the noise level observed in the recorded $\mathrm{m}(\mathrm{T})$ curve, above $\mathrm{T}_{C}$. 
Finally, we stress that more than 10 hybrids have been examined and all exhibited consistent behavior regarding the piezoelectric modification of $\mathrm{T}_{C}$ upon application of strain, $\mathrm{S}\left(\mathrm{E}_{e x}\right)$, through the external electric field, $\mathrm{E}_{e x}$.

\section{EXPERIMENTAL RESULTS AND PHENOMENOLOGICAL MODELING}

Figure 1(a) shows a photographic illustration of an $\mathrm{Nb} /$ $\mathrm{PMN}-\mathrm{xPT} / \mathrm{Nb}$ hybrid. The two $\mathrm{Nb}$ thin films extend over the entire large surfaces of the PMN-xPT single crystal that after production was left unpoled. In this configuration, the $\mathrm{Nb}$ outer layers serve as electrodes for the application of the external voltage, $\mathrm{V}_{e x}$, that produces the external electric field, $\mathrm{E}_{e x}$, to the PMN-xPT single crystal. A schematic coordinate system is also shown to specify, first, the directions where the external electric, $\mathrm{E}_{e x}$ and magnetic, $\mathrm{H}_{e x}$ fields are applied (out-of-plane and in-plane, respectively) and, second, the electro-mechanical response of the PMN-xPT single crystals upon application of $\mathrm{E}_{e x}$. It is expected that the strain produced by the PMN-xPT single crystal will distort the crystal lattice of the $\mathrm{Nb}$ thin films. A schematic illustration of a SC/PE/SC hybrid is shown in Figs. 1(b) and 1(c) to demonstrate the influence of the in-plane electro-mechanical response of the PE interlayer on the crystal structure of the SC outer layers. Specifically, under the application of an out-of-plane $\mathrm{E}_{e x}$ (that is along the $\mathrm{z}$ axis), we can modify the lattice constants of the PE single crystal from $\mathrm{a}_{0}, \mathrm{~b}_{0}$, and $\mathrm{c}_{0}$ for $\mathrm{E}_{e x}=0$, to $\mathrm{a}_{0}^{/}, \mathrm{b}_{0}^{/}$, and $\mathrm{c}_{0}^{l}$ for $\mathrm{E}_{e x} \neq 0$. Following the in-plane electro-mechanical response of the PE interlayer that is expansive along $\mathrm{x}$ and $\mathrm{z}$ axes and compressive along the $y$-axis, the lattice constants of the SC outer layers are modified accordingly, from $\mathrm{a}, \mathrm{b}$, and $\mathrm{c}$ for $\mathrm{E}_{e x}=0$ to $\mathrm{a}^{/}>\mathrm{a}, \mathrm{b}^{\prime}<\mathrm{b}$, and $\mathrm{c}^{/}>\mathrm{c}$. This would ultimately affect the critical temperature, $\mathrm{T}_{C}$.

\section{A. Experimental results}

Figures 2(a.i)-2(a.iii), 2(b.i)-2(b.iii), and 2(c.i)-2(c.iii) show three sets of magnetization data, obtained at low values of $\mathrm{H}_{e x}$, for three different $\mathrm{Nb} / \mathrm{PMN}-\mathrm{xPT} / \mathrm{Nb}$ hybrids. These
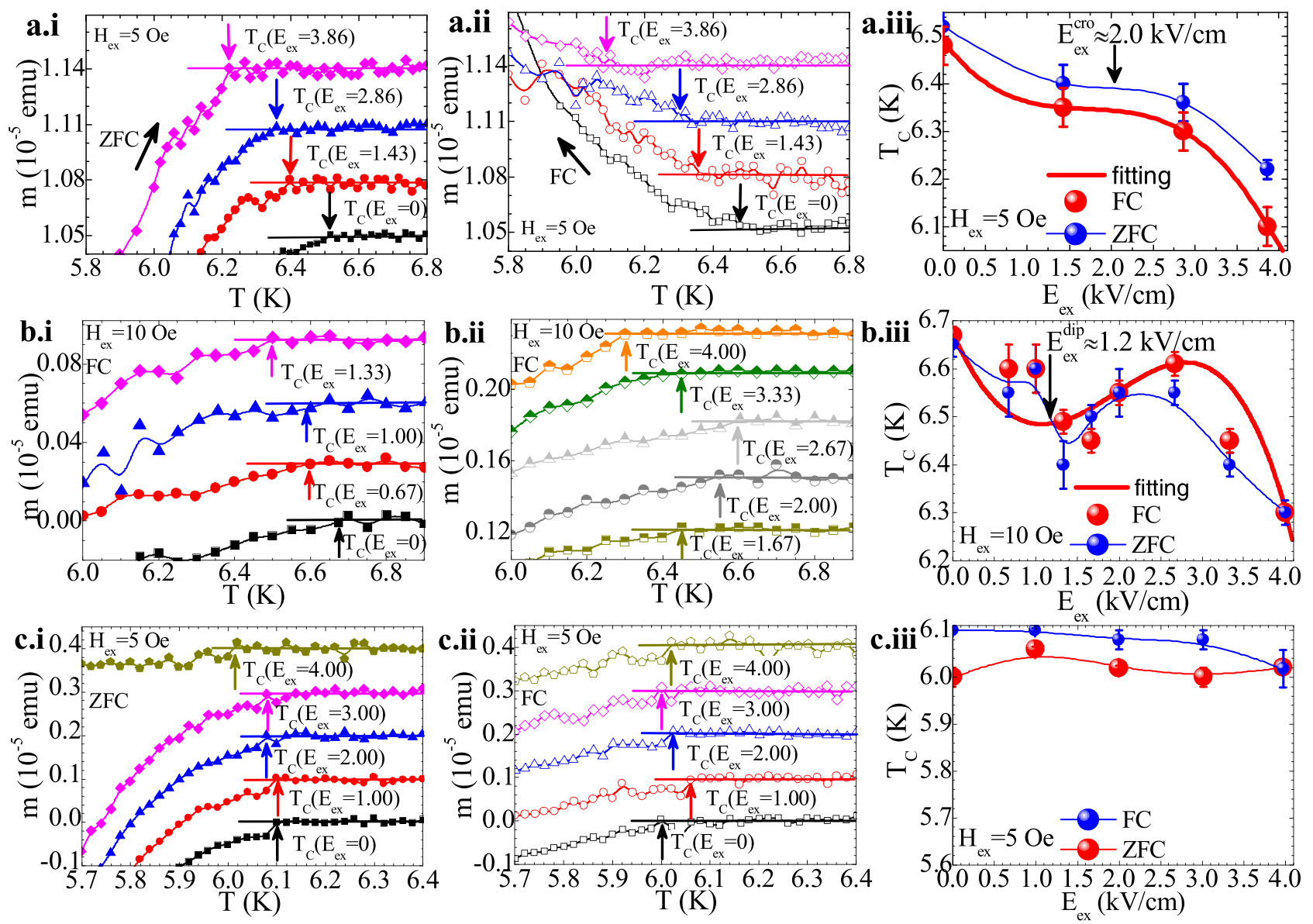

FIG. 2. (a.i)-(a.iii) Polished PMN-xPT single crystal $(\mathrm{Sa}=27 \mathrm{~nm})$ with $\mathrm{x}=0.31$, while $\mathrm{d}_{N b}=15 \mathrm{~nm}$; data obtained at $\mathrm{H}_{e x}=5$ Oe. (a.i) and (a.ii) Isofield magnetization data, $\mathrm{m}(\mathrm{T})$ in a reduced temperature range, for both (a.i) zero-field-cooling (ZFC) and (a.ii) field-cooling (FC) branches [m(T) curves are vertically shifted for clarity]. (a.iii) Summary of $\mathrm{T}_{C}\left(\mathrm{E}_{e x}\right)$ for both the ZFC (blue spheres) and FC (red spheres) case, together with a fitting curve (thick-solid-red line) for the FC data (see Sec. IV of the supplementary material). A crossover is observed at $\mathrm{E}_{e x}^{\text {cro }} \approx 2.0 \mathrm{kV} / \mathrm{cm}$. (b.i)-(b.iii) Non-polished PMN-xPT single crystal $\left(\mathrm{Sa}=218 \mathrm{~nm}\right.$ ) with $\mathrm{x}=0.31$, while $\mathrm{d}_{N b}=20 \mathrm{~nm}$; data obtained at $\mathrm{H}_{e x}=10 \mathrm{Oe}$. (b.i) and (b.ii) Isofield magnetization data, m(T) for the $\mathrm{FC}$ mode, in a reduced temperature range, for (b.i) low and (b.ii) high values of $\mathrm{E}_{e x}\left[\mathrm{~m}(\mathrm{~T})\right.$ curves are vertically shifted for clarity]. (b.iii) Summary of $\mathrm{T}_{C}\left(\mathrm{E}_{e x}\right)$ for both the $\mathrm{ZFC}$ (blue spheres) and FC (red spheres) case, together with a fitting curve (thick-solid-red line) for the FC data (see the text for details). A pronounced dip is observed at $\mathrm{E}_{e x}^{d i p} \approx 1.2 \mathrm{kV} / \mathrm{cm}$. (c.i)-(c.iii) Polished PMN-xPT single crystal $\left(\mathrm{Sa}=23 \mathrm{~nm}\right.$ ) with $\mathrm{x}=0.27$, while $\mathrm{d}_{N b}=15 \mathrm{~nm}$; data obtained at $\mathrm{H}_{e x}=5 \mathrm{Oe}$. (c.i) and (c.ii) Isofield magnetization data, $\mathrm{m}(\mathrm{T})$ in a reduced temperature range, for both (c.i) ZFC and (c.ii) FC branches [m(T) curves are vertically shifted for clarity]. (c.iii) Summary of $\mathrm{T}_{C}\left(\mathrm{E}_{e x}\right)$ for both ZFC (blue spheres) and FC (red spheres) branches. The blue and red thin lines serve as a guide to the eye. In all panels (a.iii)-(c.iii), the horizontal (electric field) and the vertical (temperature) scales are the same. 
data are grouped with respect to the characteristics of each PMN-xPT single crystal. Figures 2(a.i)-2(a.iii) refer to a polished PMN-xPT single crystal $(\mathrm{Sa}=27 \mathrm{~nm})$ with $\mathrm{x}=0.31$ $\left(\mathrm{d}_{N b}=15 \mathrm{~nm}\right)$. Figures 2(b.i)-2(b.iii) refer to a non-polished PMN-xPT single crystal $(\mathrm{Sa}=218 \mathrm{~nm})$ with the same $\mathrm{x}=0.31\left(\mathrm{~d}_{N b}=20 \mathrm{~nm}\right)$. Finally, Figs. 2(c.i)-2(c.iii) refer to a polished PMN-xPT single crystal $(\mathrm{Sa}=23 \mathrm{~nm})$, however, with different $\mathrm{x}=0.27\left(\mathrm{~d}_{N b}=15 \mathrm{~nm}\right)$. Figures 2(a.i)-2(a.iii) show the $\mathrm{m}(\mathrm{T})$ curves for both zero-field-cooling (ZFC) and field-cooling (FC) modes in Figs. 2(a.i) and 2(a.ii), respectively. The paramagnetic behavior observed only in the FC branch in some of the studied hybrids [e.g., Fig. 2(a.ii)] is a well-known effect that appears in both low/high- $\mathrm{T}_{C} \mathrm{SC}$ films and superlattices/composites of $\mathrm{SC}$ with magnetic/normal metals. ${ }^{33-36}$ Figure 2(a.iii) summarizes the $\mathrm{T}_{C}\left(\mathrm{E}_{e x}\right)$ data for both the ZFC and FC case, together with a fitting curve for the FC data (see Sec. IV of the supplementary material). We see that $\mathrm{T}_{C}$ progressively decreases upon increase of $\mathrm{E}_{e x}$, while a crossover is evident at $\mathrm{E}_{e x}^{c r o} \approx 2.0 \mathrm{kV} / \mathrm{cm}$. Figures 2(b.i)-2(b.iii) focus on FC $\mathrm{m}(\mathrm{T})$ curves obtained upon systematic variation of $\mathrm{E}_{e x}$ to refine the character of the crossover observed in Fig. 2(a.iii). These data are presented in a reduced temperature range, for low and high values of $\mathrm{E}_{e x}$ in Figs. 2(b.i) and 2(b.ii), respectively. Figure 2(b.iii) summarizes the $\mathrm{T}_{C}\left(\mathrm{E}_{e x}\right)$ data for both the $\mathrm{ZFC}$ and $\mathrm{FC}$ case, together with a fitting curve for the FC data (see below). These detailed data unveil the additional characteristics in the regime of intermediate values of $\mathrm{E}_{e x}$, where a pronounced dip is now clearly evident at $\mathrm{E}_{e x}^{d i p} \approx 1.2 \mathrm{kV} / \mathrm{cm}$. Finally, Figs. 2(c.i)-2(c.iii) present both ZFC, Fig. 2(c.i), and FC, Fig. 2(c.ii), data in a reduced temperature range, while, Fig. 2(c.iii) shows the $\mathrm{T}_{C}\left(\mathrm{E}_{e x}\right)$ data for both cases.

The data of Figs. 2(a.iii)-2(c.iii) are summarized as follows. For the polished PMN-xPT single crystal with $\mathrm{x}=0.31$ [Fig. 2(a.iii)], the overall decrease of $\mathrm{T}_{C}\left(\mathrm{E}_{e x}\right)$ for $\mathrm{E}_{\max }$ $=4 \mathrm{kV} / \mathrm{cm}$ is approximately $400 \mathrm{mK}$ that corresponds to a modification coefficient (percentage) of $0.100 \mathrm{~K} / \mathrm{kV} / \mathrm{cm}$ (6.0\%). The situation is almost the same for the non-polished PMN-xPT single crystal with the same $x=0.31$ [Fig. 2(b.iii)]. On the contrary, for polished PMN-xPT, but with $\mathrm{x}=0.27$ [Fig. 2(c.iii)], $\mathrm{T}_{C}\left(\mathrm{E}_{e x}\right)$ exhibits a minor decrease (if any) only in the ZFC branch with the modification coefficient (percentage) $0.020 \mathrm{~K} / \mathrm{kV} / \mathrm{cm}(1.3 \%)$. This is approximately 5 times lower than that observed for PMN-xPT single crystals with $\mathrm{x}=0.31$, whether polished [Fig. 2(a.iii)] or non-polished [Fig. 2(b.iii)].

Accordingly, two safe conclusions can be drawn. First, the overall modification of $\mathrm{T}_{C}$ by $\mathrm{E}_{e x}$ is not seriously affected by the surface roughness of the PMN-xPT single crystals. Though someone could invoke that the extrinsic factor of surface roughness motivates the development of the weak crossover observed in Fig. 2(a.iii) (polished PMN-xPT with $\mathrm{Sa}=27 \mathrm{~nm}$ ) and of the pronounced dip observed in Fig. 2(b.iii) (non-polished PMN-xPT with $\mathrm{Sa}=218 \mathrm{~nm}$ ), below we show that both features reflect an intrinsic property of PMN-xPT single crystals with $\mathrm{x}=0.31$ originating from their electro-mechanical response upon application of $\mathrm{E}_{e x}$. Nevertheless, the data of Figs. 2(a.iii) and 2(b.iii) suggest that increased surface roughness can probably promote this feature (see Sec. V of the supplementary material). Second, the modification of $\mathrm{T}_{C}$ by $\mathrm{E}_{e x}$ is improved drastically when PMN-xPT single crystals with PT content near the morphotropic phase boundary $(\mathrm{x}=0.31)$ are used since they exhibit maximum electric-field-induced strain, $S\left(\mathrm{E}_{e x}\right){ }^{28}$

The experimental data on $\mathrm{T}_{C}\left(\mathrm{E}_{e x}\right)$ of the $\mathrm{Nb}$ thin films can be reproduced with a phenomenological model by using relation (1) when the electric-field-induced strain of the PMN-xPT single crystals is taken into account through the respective constitutive relation of the "Strain-Electric field" curve, $S\left(\mathrm{E}_{e x}\right)$. Accordingly, in Figs. 3(a) and 3(b) we introduce raw experimental data on the in-plane compressive strain $\mathrm{S}_{2}\left(\mathrm{E}_{e x, 3}\right)$ for a PMN-xPT single crystal with $\mathrm{x}=0.31$. The data were obtained with a global $[\text { Fig. } 3(\mathrm{a})]^{28}$ and a local [Figs. 3(b) and 3(c) $]^{30,31}$ technique and are focused in the regime of low $\mathrm{E}_{e x, 3}$ for the sake of comparison with the $\mathrm{T}_{C}\left(\mathrm{E}_{e x}\right)$ data of Figs. 2(a.iii) and 2(b.iii). Both techniques reveal an anomaly in the $\mathrm{S}_{2}\left(\mathrm{E}_{e x, 3}\right)$; an inflection point, $\mathrm{E}_{e x, 3}^{i n f}$, where a change in the concavity is evidenced in the global data [Fig. 3(a); $\mathrm{E}_{e x, 3}^{i n f} \approx 1.4 \mathrm{kV} / \mathrm{cm}$ ], accompanied by a weak, however discernible, anomaly, $\mathrm{E}_{e x, 3}^{i n f}$, in the local data [Figs. 3 (b) and $\left.3(\mathrm{c}) ; \mathrm{E}_{e x, 3}^{i n f} \approx 1.2 \mathrm{kV} / \mathrm{cm}\right]$. Furthermore, a more pronounced change in the slope, $\mathrm{E}_{e x, 3}^{c h a}$, is observed in the local data [Fig. 3(b); $\mathrm{E}_{e x, 3}^{c h a} \approx 2.0 \mathrm{kV} / \mathrm{cm}$ ]. These features are probably motivated by an electric-field induced rhombohedral to orthorhombic phase transition taking place at $2 \mathrm{kV} / \mathrm{cm}^{28,30}$
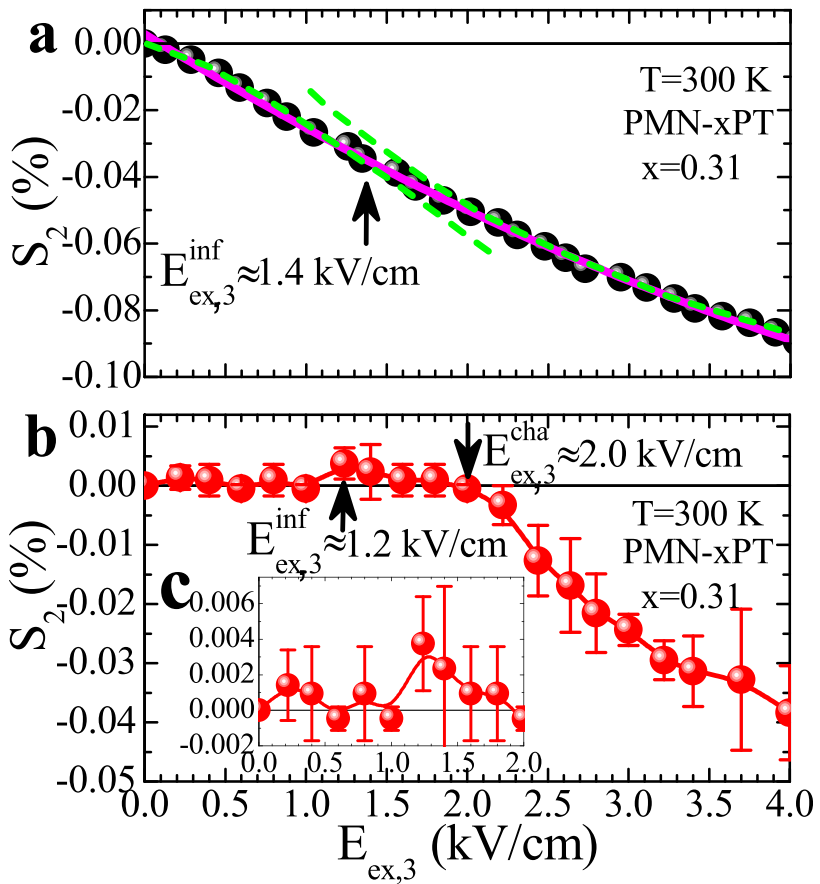

FIG. 3. (a)-(c) Raw in-plane compressive $S_{2}\left(E_{e x, 3}\right)$ experimental data for a PMN-xPT crystal with $\mathrm{x}=0.31$, for low values of $\mathrm{E}_{e x, 3}$, obtained via (a) a global $^{28}$ and (b), (c) a local ${ }^{30,31}$ technique. (a) A relatively weak inflection point is observed in the $\mathrm{S}_{2}\left(\mathrm{E}_{e x, 3}\right)$ global experimental data at $\mathrm{E}_{e x, 3}^{i n f} \approx 1.4 \mathrm{kV} /$ $\mathrm{cm}$ and is highlighted by the divergence of the green-dashed curves that serve as a guide to the eye. The magenta-solid line refers to second order fitting of the $\mathrm{S}_{2}\left(\mathrm{E}_{e x, 3}\right)$ global experimental data. (b) A pronounced change in the slope is observed in the $\mathrm{S}_{2}\left(\mathrm{E}_{e x, 3}\right)$ local experimental data at $\mathrm{E}_{e x, 3}^{c h a} \approx 2.0$ $\mathrm{kV} / \mathrm{cm}$. (c) The inset focuses on the clear anomaly observed in the local data, at the same point where the weak inflection point is observed in the global ones, panel (a). In both cases, $\mathrm{S}_{2}$ refers to $\mathrm{S}_{y}$, while $\mathrm{E}_{e x}$ refers to $\mathrm{E}_{e x, 3}$; see Figs. 1(a)-1(c). 
The noticeable difference between the local and global measurements is due to the fact that the PMN-xPT solid solution shows compositional inhomogeneity mainly attributed to the Ti segregation. ${ }^{28,30}$ Accordingly, a small electric field on the order of $2 \mathrm{kV} / \mathrm{cm}$, can induce a large strain in specific areas of the crystals with higher PT content, as indeed recorded with the local technique. On the contrary, the global technique records the average response over the entire sample so that any abrupt feature is smoothed out. Impressively, a clear anomaly is also observed in the local data, Fig. 3(c), at the same point where the weak inflection point is observed in the global ones, Fig. 3(a). Clearly, both points coincide fairly with the crossover and the dip observed at $\mathrm{E}_{e x}^{c r o} \approx 2.0 \mathrm{kV} /$ $\mathrm{cm})$ and $\left.\mathrm{E}_{e x}^{\text {dip }} \approx 1.2 \mathrm{kV} / \mathrm{cm}\right)$, respectively, in the $\mathrm{T}_{C}\left(\mathrm{E}_{e x}\right)$ data of the relevant $\mathrm{Nb} / \mathrm{PMN}-\mathrm{xPT} / \mathrm{Nb}$ hybrids [Figs. 2(a.iii) and 2(b.iii)]. These combined data prove that the critical temperature, $\mathrm{T}_{C}$ depends on the in-plane compressive strain, $\mathrm{S}_{2}\left(\mathrm{E}_{e x, 3}\right)$.

\section{B. Phenomenological modeling}

Let us now introduce the phenomenological model by comparing the $\mathrm{S}_{2}\left(\mathrm{E}_{e x, 3}\right)$ data of PMN-xPT single crystals with $\mathrm{x}=0.31$, Fig. 3(a), with the $\mathrm{T}_{C}\left(\mathrm{E}_{e x}\right)$ data of the relevant $\mathrm{Nb} / \mathrm{PMN}-\mathrm{xPT} / \mathrm{Nb}$ hybrids with the same PT content $\mathrm{x}=0.31$. Specifically, we test the phenomenological model for the FC data of the hybrid of Fig. 2(b.iii) [for the respective FC data of the hybrid of Fig. 2(a.iii) see Sec. IV of the supplementary material]. Quantitatively, we proceed by employing the $\mathrm{S}_{2}\left(\mathrm{E}_{e x, 3}\right)$ data obtained on PMN-xPT single crystals via the global technique, since the relevant $\mathrm{T}_{C}\left(\mathrm{E}_{e x}\right)$ data of the respective $\mathrm{Nb} / \mathrm{PMN}-\mathrm{xPT} / \mathrm{Nb}$ hybrids were obtained via global magnetization measurements, as well. Various functions can be used to fit the experimental data $\mathrm{S}_{2}\left(\mathrm{E}_{e x, 3}\right)$ of Fig. 3(a). Here, for the sake of clarity, we use a minimal second order constitutive relation

$$
S\left(E_{e x}\right)=A+B E_{e x}+C E_{e x}^{2},
$$

that reproduces the behavior of the $\mathrm{S}_{2}\left(\mathrm{E}_{\text {ex,3 }}\right)$ with $\mathrm{A}=2.82$ $\times 10^{-3} \%$ and $\mathrm{B}=-29.90 \times 10^{-3} \% / \mathrm{kV} / \mathrm{cm}$ and $\mathrm{C}=1.75$ $\times 10^{-3} \% /(\mathrm{kV} / \mathrm{cm})^{2}$ [magenta-solid line in Fig. 3(a)] with great accuracy, including the inflection point observed at $\mathrm{E}_{e x, 3}^{i n f} \approx 1.4 \mathrm{kV} / \mathrm{cm}$. Accordingly, as shown below, the model performs excellently [for the hybrid of Fig. 2(a.iii), a more simple linear constitutive relation, $S\left(\mathrm{E}_{e x}\right)$ is only needed; see Sec. IV of the supplementary material].

To fit the FC experimental data of $\mathrm{T}_{C}\left(\mathrm{E}_{e x}\right)$, Fig. 2(b.iii), in the context of the well established theoretical relation (1), we employ a phenomenological model according to which all involved parameters $\lambda, \mu^{*}$, and $\omega_{l n}$, relation (1), depend indirectly on $\mathrm{E}_{e x}$, through their primary dependence on strain, $\mathrm{S}$, that is through the constitutive relation $S\left(\mathrm{E}_{e x}\right)$. For the dependence of $\lambda, \mu^{*}$ and $\omega_{l n}$ on $\mathrm{S}$ various trial functions can be used. Here, for $\lambda$ and $\mu^{*}$ we choose a rather steep, exponential, function, $\lambda(\mathrm{S})=\lambda_{0} \exp \left(-\lambda_{1} \mathrm{~S}\right)$ and $\mu^{*}(\mathrm{~S})=\mu_{0}^{*} \exp \left(+\mu_{1}^{*} \mathrm{~S}\right)$ since in the framework of the BCS theory, the parameters $\lambda$ and $\mu^{*}$ are allowed to vary only within a specifically narrow range, usually $0.5<\lambda<2.0$ and $0.1<\mu^{*}<0.2$; $^{25-27,37-39}$ else, superconductivity is suppressed rapidly upon even small deviations of $\lambda$ and $\mu^{*}$. In these relations, parameters $\lambda_{0}$ and $\mu_{0}^{*}$ refer to the unperturbed attractive and repulsive potentials of the undistorted crystal lattice $(\mathrm{S}=0)$, while exponents $\lambda_{1}$ and $\mu_{1}^{*}$ determine the magnitude of the distortion imposed to the crystal lattice, thus termed distortion coefficients. For the specific set of FC data shown in Fig. 2(b.iii), the choice of the distortion coefficients $-\lambda_{1}<0$ and $+\mu_{1}^{*}>0$ is based on the physical requisite that the phonon-mediated electron-electron attractive potential, $\lambda$, should decrease with $\mathrm{S}$, while the Coulomb electron-electron repulsive potential, $\mu^{*}$, should increase with $\mathrm{S}$. These requisites conform to the physical origin of relation (1), ${ }^{24-27}$ when used to describe the decrease of $\mathrm{T}_{C}$ by any cause. For the dependence of $\omega_{l n}$ on $\mathrm{S}$, we choose a more smooth, however, non-linear, function, $\omega_{l n}(\mathrm{~S})=\omega_{l n, 0}$ $+\omega_{l n, 1} \mathrm{~S}+\omega_{l n, 2} \mathrm{~S}^{2}$, where the parameter $\omega_{l n, 0}$ refers to the unperturbed mean frequency of phonons $(S=0)$, while parameters $\omega_{l n, 1}$ and $\omega_{l n, 2}$ are the respective distortion coefficients During the fittings, $\omega_{\ln , 1}$ and $\omega_{l n, 2}$ are left free to vary, having no inherent predisposition on $\mathrm{S}$ (thus, both stiffening and softening of the phonon branches ${ }^{40,41}$ can be taken into account).

Incorporating the constitutive relation (2) in the above relations for $\lambda(\mathrm{S}), \mu^{*}(\mathrm{~S})$ and $\omega_{l n}(\mathrm{~S})$, respectively, and substituting into relation (1), we get the phenomenological relation

$$
\begin{aligned}
\mathrm{T}_{C}\left(\mathrm{E}_{e x}\right)= & \left(\omega_{l n}\left(\mathrm{E}_{e x}\right) / 1.20\right) \\
& \times \exp \left(-\frac{1.04\left(1+\lambda\left(E_{e x}\right)\right)}{\lambda\left(E_{e x}\right)-\mu^{*}\left(E_{e x}\right)\left(1+0.62 \lambda\left(E_{e x}\right)\right)}\right),
\end{aligned}
$$

where

$$
\begin{aligned}
\lambda\left(E_{e x}\right) & =\lambda_{0}^{\prime} \exp \left(-\left(\lambda_{1}^{\prime} E_{e x}+\lambda_{2}^{\prime} E_{e x}^{2}\right)\right), \\
\mu^{*}\left(E_{e x}\right) & =\mu_{0}^{* /} \exp \left(+\mu_{1}^{* /} E_{e x}+\mu_{2}^{* /} E_{e x}^{2}\right),
\end{aligned}
$$

and

$$
\omega_{l n}\left(E_{e x}\right)=\omega_{l n, 0}^{\prime}+\omega_{l n, 1}^{\prime} E_{e x}+\omega_{l n, 2}^{\prime} E_{e x}^{2}+\omega_{l n, 3}^{\prime} E_{e x}^{3}+\omega_{l n, 4}^{\prime} E_{e x}^{4} .
$$

The relation between the newly introduced primed parameters $\lambda_{0}^{\prime}, \lambda_{1}^{\prime}, \lambda_{2}^{\prime}, \mu_{0}^{* \prime}, \mu_{1}^{* /}, \mu_{2}^{* \prime}, \omega_{l n, 0}^{\prime}, \omega_{l n, 1}^{\prime}, \omega_{l n, 2}^{\prime}, \omega_{l n, 3}^{\prime}$, and $\omega_{l n, 4}^{/}$with the former, non-primed, ones is given in Table I.

By using relation (3), we fitted the $\mathrm{T}_{C}\left(\mathrm{E}_{e x}\right) \mathrm{FC}$ experimental data of Fig. 2(b.iii) (fitting curve; red thick line). The obtained primed fitting parameters are given in Table II. A number of conclusions can be drawn from the approach employed here. First, we stress that all unperturbed fitting parameters of Table II $\left(\lambda_{0}^{\prime}, \mu_{0}^{* /}\right.$, and $\left.\omega_{l n, 0}^{\prime}\right)$ range within reasonable limits. ${ }^{6,26,27,37-39}$ Second, quantitatively, all distortion coefficients of Table II $\left(\lambda_{1}^{l}, \lambda_{2}^{l}, \mu_{1}^{* /}, \mu_{2}^{* /}, \omega_{l n, 1}^{l}, \omega_{l n, 2}^{l}\right.$, $\omega_{l n, 3}^{\prime}$, and $\omega_{l n, 4}^{\prime}$ ) obtain reasonable values, as well. Third, as a consequence of these facts, all attractive potential $\lambda\left(\mathrm{E}_{e x}\right)$ [relation (4)], repulsive potential $\mu^{*}\left(\mathrm{E}_{e x}\right)$ [relation (5)], and mean frequency of phonons $\omega_{l n}\left(\mathrm{E}_{e x}\right)$ [relation (6)], exhibit a 
TABLE I. Relation between non-primed and primed parameters entering relations $\lambda\left(\mathrm{E}_{e x}\right), \mu^{*}\left(\mathrm{E}_{e x}\right)$ and $\omega_{l n}\left(\mathrm{E}_{e x}\right)$. Parameters $\mathrm{A}, \mathrm{B}$, and $\mathrm{C}$ are fixed to the values obtained while using the second order constitutive relation $\mathrm{S}\left(\mathrm{E}_{e x}\right)$ of the PE single crystal, relation (2), to fit the global experimental data $\mathrm{S}_{2}\left(\mathrm{E}_{e x, 3}\right)$, Fig. 3(a).

\begin{tabular}{lcc}
\hline \hline$\lambda\left(\mathrm{E}_{e x}\right)$ & $\mu^{*}\left(\mathrm{E}_{e x}\right)$ & $\omega_{l n}\left(\mathrm{E}_{e x}\right)$ \\
\hline$\lambda_{0}^{\prime}=\lambda_{0} \exp \left(-\lambda_{1} \mathrm{~A}\right)$ & $\mu_{0}^{* \prime}=\mu_{0}^{*} \exp \left(+\mu_{1}^{*} \mathrm{~A}\right)$ & $\omega_{l n, 0}^{\prime}=\omega_{l n, 0}+\omega_{l n, 1} \mathrm{~A}+\omega_{l n, 2} A^{2}$ \\
$\lambda_{1}^{\prime}=\lambda_{1} \mathrm{~B}$ & $\mu_{1}^{* \prime}=\mu_{1}^{*} \mathrm{~B}$ & $\omega_{l n, 1}^{\prime}=\omega_{l n, 1} \mathrm{~B}+\omega_{l n, 2} 2 \mathrm{AB}$ \\
$\lambda_{2}^{\prime}=\lambda_{1} \mathrm{C}$ & $\mu_{2}^{* \prime}=\mu_{1}^{*} \mathrm{C}$ & $\omega_{l n, 2}^{\prime}=\omega_{l n, 1} \mathrm{C}+\omega_{l n, 2}\left(\mathrm{~B}^{2}+2 \mathrm{AC}\right)$ \\
& & $\omega_{l n, 3}^{\prime}=\omega_{l n, 2} \mathrm{BC}$ \\
& & $\omega_{l n, 4}=\omega_{l n, 2} C^{2}$ \\
\hline \hline
\end{tabular}

sensible behavior ${ }^{6,26,27,37-39}$ in the entire range of $\mathrm{E}_{e x}$, where the FC experimental data of $\mathrm{T}_{C}\left(\mathrm{E}_{e x}\right)$ lie [Fig. 2(b.iii)]. This is summarized in Fig. 4. We believe that the latter fact proves the self-consistency of the overall experimentalphenomenological modeling approach introduced here. Thanks to its flexibility, the present phenomenological model can be adjusted accurately to the specific characteristics of both SC and PE ingredients, thus it can describe successfully the $\mathrm{T}_{C}\left(\mathrm{E}_{e x}\right.$ behavior of virtually any $\mathrm{SC} / \mathrm{PE}$ hybrid$)$.

\section{Perspectives}

Recently, in Ref. 42, the authors studied a field-effect transistor device consisting of a thin single crystal of organic $\kappa-(\mathrm{BEDT}-\mathrm{TTF})_{2} \mathrm{Cu}\left[\mathrm{N}(\mathrm{CN})_{2}\right] \mathrm{Cl}$ developed onto flexible substrates of polyethylene naphtalate. Strain was applied to the device by mechanical means and the resistance was recorded as a function of either temperature (iso-strain curves) or strain up to $1.1 \%$ (iso-thermal curves). In both cases, the authors observed a strong resistance reduction of approximately 8 orders of magnitude, proving that the device switches between the superconducting and normal states. In the $\mathrm{Nb} / \mathrm{PMN}-\mathrm{xPT} / \mathrm{Nb}$ hybrids studied here, we observed a modification of $\mathrm{T}_{C} \approx 0.5 \mathrm{~K}$ that is a percentage modulation $\approx 6.0 \%$. This means that, yet, much is to be done regarding the absolute control of superconductivity (i.e., $100 \%$ modification of $\mathrm{T}_{C}$ ) of a low- $\mathrm{T}_{C}$ superconductor, through electricfield-induced strain. Nevertheless, our work adds qualitatively new and quantitatively noticeable information to the field of SC/PE hybrids that seems to be relatively stagnant when compared to the achievements reported for ferromagnetic/PE ones. For instance, in Ref. 20 the authors investigated the high- $\mathrm{T}_{C}$ superconductor $\mathrm{YBa}_{2} \mathrm{Cu}_{3} \mathrm{O}_{7-x}$ in relative $\mathrm{PMN}-0.28 \mathrm{PT} / \mathrm{SrRuO}_{3} / \mathrm{YBa}_{2} \mathrm{Cu}_{3} \mathrm{O}_{7-x}$ hybrids and reported a modulation of $\mathrm{T}_{C} \approx 0.07 \mathrm{~K}$ for a voltage variation of $350 \mathrm{~V}$

TABLE II. Primed fitting parameters of relations (3) and (4)-(6), used to reproduce the FC data of Fig. 2(b.iii). During the fitting, all parameters are fixed to the specified values.

\begin{tabular}{lcc}
\hline \hline$\lambda\left(\mathrm{E}_{e x}\right)$ & $\mu^{*}\left(\mathrm{E}_{e x}\right)$ & $\omega_{\ln }\left(\mathrm{E}_{\text {ex }}\right)$ \\
\hline$\lambda_{0}^{\prime}=1.503$ & $\mu_{0}^{* /}=0.105$ & $\omega_{\ln , 0}^{\prime}=65.454$ \\
$\lambda_{1}^{\prime}=0.085$ & $\mu_{1}^{* /}=0.025$ & $\omega_{\ln , 1}^{\prime}=-8.725$ \\
$\lambda_{2}^{\prime}=0.005$ & $\mu_{2}^{* /}=0.005$ & $\omega_{\ln , 2}^{\prime}=2.825$ \\
& & $\omega_{\ln , 3}^{\prime}=-0.485$ \\
& & $\omega_{\ln , 4}^{l}=0.008$ \\
\hline \hline
\end{tabular}

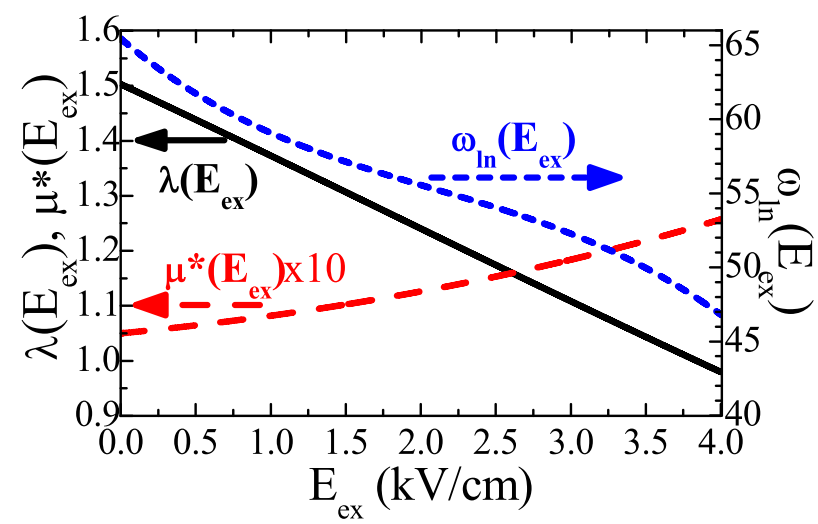

FIG. 4. Variation of the attractive potential, $\lambda\left(\mathrm{E}_{e x}\right)$, repulsive potential, $\mu^{*}\left(\mathrm{E}_{e x}\right)$, and mean frequency of phonons, $\omega_{l n}(E e x)$, in the range of low values of $\mathrm{E}_{e x}, 0.0 \mathrm{kV} / \mathrm{cm} \leq \mathrm{E}_{e x} \leq 4.0 \mathrm{kV} / \mathrm{cm}$, where the $\mathrm{FC}$ experimental data, $\mathrm{T}_{C}\left(\mathrm{E}_{e x}\right)$, of Fig. 2(b.iii) lie. Notice that $\mu^{*}\left(\mathrm{E}_{e x}\right)$ is multiplied with a factor of 10 for the sake of comparative presentation.

(electric field variation $\approx 8.4 \mathrm{kV} / \mathrm{cm}$ ). Due to the high- $\mathrm{T}_{C}$ of $\mathrm{YBa}_{2} \mathrm{Cu}_{3} \mathrm{O}_{7-x}$, this implies a modest percentage modulation of $\mathrm{T}_{C} \approx 8 \times 10^{-3} \%$. Also, in Ref. 21 the authors studied PMN-0.28PT combined with other high- $\mathrm{T}_{C}$ materials, namely, $\mathrm{La}_{1.85} \mathrm{Sr}_{0.15} \mathrm{CuO}_{4}$ and $\mathrm{BaFe}_{1.8} \mathrm{Co}_{0.2} \mathrm{As}_{2}$. They reported a modulation of $\mathrm{T}_{C}$ on the order of $0.4 \mathrm{~K}$ (percentage modulation $\approx 2.3 \%$ ) and $0.2 \mathrm{~K}$ (percentage modulation $\approx 1.4 \%$ ), respectively, under the application of an electric field $\mathrm{E}_{e x} \approx 10 \mathrm{kV} / \mathrm{cm}$. Still, it is not clear whether the modest progress in the field of SC/PE stems from endogenous restrictions (e.g., generic incompatibility of the underlying mechanisms) or from exogenous complications (e.g., not appropriately combined constituents). In this regard, the experimental data and phenomenological modeling presented here could assist toward understanding the class of SC/PE hybrids.

\section{CONCLUSION}

In conclusion, controlled modification of the critical temperature, $\mathrm{T}_{C}$, by means of electric-field-induced strain, $\mathrm{S}\left(\mathrm{E}_{e x}\right)$, was evidenced experimentally in $\mathrm{Nb} / \mathrm{PMN}-\mathrm{xPT} / \mathrm{Nb}$ hybrids $\left(\mathrm{x}=0.27\right.$ and $\left.0.31,3 \mathrm{~nm} \leq \mathrm{d}_{N b} \leq 50 \mathrm{~nm}\right)$ for PMNPT crystals of different surface roughness. A conceptually consistent and practically concise phenomenological model was employed to successfully describe the experimental data; due to its easy-to-apply and practical character, it can be used as a general recipe and be applied in any SC/PE hybrid.

\section{SUPPLEMENTARY MATERIAL}

See supplementary material for details on the experimental techniques and extra sets of both experimental and fitting data.

\section{ACKNOWLEDGMENTS}

One of the authors (M.Z.) acknowledges the A.G. Leventis Foundation for support through a scholarship. The authors acknowledge Dr. Jun Luo at TRS Technologies for offering part of the PMN-xPT crystals. 
${ }^{1}$ G. Deutscher and F. Meunier, Phys. Rev. Lett. 22, 395 (1969).

${ }^{2}$ N. M. Nemes, C. Visani, C. Leon, M. Garcia-Hernandez, F. Simon, T. Fehér, S. G. E. te Velthuis, A. Hoffmann, and J. Santamaria, Appl. Phys. Lett. 97, 032501 (2010).

${ }^{3}$ I. F. Lyuksyutov and V. L. Pokrovsky, "Ferromagnet-superconductor hybrids," Adv. Phys. 54, 67 (2005).

${ }^{4}$ D. Stamopoulos and E. Aristomenopoulou, Sci. Rep. 5, 13420 (2015).

${ }^{5}$ J. L. Olsen, E. Bucher, M. Levy, J. Muller, E. Corenzwit, and T. Geballe, Rev. Mod. Phys. 36, 168 (1964).

${ }^{6}$ V. V. Struzhkin, Y. A. Timofeev, R. J. Hemley, and H.-k. Mao, Phys. Rev. Lett. 79, 4262 (1997).

${ }^{7}$ D. Gu, Q. Wu, Y. Zhou, P. Gao, J. Guo, C. Zhang, S. Zhang, S. Jiang, K. Yang, and A. Li, New J. Phys. 17, 073021 (2015).

${ }^{8}$ J. W. Ekin, Cryogenics 20, 611 (1980).

${ }^{9}$ D. C. van der Laan, J. W. Ekin, J. F. Douglas, C. C. Clickner, T. C. Stauffer, and L. F. Goodrich, Supercond. Sci. Technol. 23, 072001 (2010).

${ }^{10}$ S. Awaji, T. Suzuki, H. Oguro, K. Watanabe, and K. Matsumoto, Sci. Rep. 5, 11156 (2015).

${ }^{11}$ J. Linder and J. W. Robinson, Nat. Phys. 11, 307 (2015).

${ }^{12}$ M. Eschrig, Rep. Prog. Phys. 78, 104501 (2015).

${ }^{13}$ H. Hayakawa, N. Yoshikawa, S. Yorozu, and A. Fujimaki, Proc. IEEE 92, 1549 (2004).

${ }^{14}$ S. Polisetty, W. Echtenkamp, K. Jones, X. He, S. Sahoo, and C. Binek, Phys. Rev. B 82, 134419 (2010).

${ }^{15}$ D. Stamopoulos, M. Zeibekis, and S. J. Zhang, J. Appl. Phys. 114, 134309 (2013).

${ }^{16}$ D. Stamopoulos, M. Zeibekis, G. Vertsioti, and S. J. Zhang, J. Appl. Phys. 116, 084304 (2014).

${ }^{17}$ N. N. Phuoc and C. K. Ong, Appl. Phys. Lett. 105, 022905 (2014).

${ }^{18}$ N. N. Phuoc and C. K. Ong, J. Appl. Phys. 118, 093903 (2015).

${ }^{19}$ A. Alberca, C. Munuera, J. Azpeitia, B. Kirby, N. M. Nemes, A. M. PerezMuñoz, J. Tornos, F. J. Mompean, C. Leon, J. Santamaria, and M. GarciaHernandez, Sci. Rep. 5, 17926 (2015).

${ }^{20}$ R. Hühne, D. Okai, K. Dörr, S. Trommler, A. Herklotz, B. Holzapfel, and L. Schultz, Supercond. Sci. Technol. 21, 075020 (2008).
${ }^{21}$ S. Trommler, R. Hühne, K. Iida, P. Pahlke, S. Haindl, L. Schultz, and B. Holzapfel, New J. Phys. 12, 103030 (2010).

${ }^{22}$ H. M. Yamamoto, M. Nakano, M. Suda, Y. Iwasa, M. Kawasaki, and R. Kato, Nat. Commun. 4, 2379 (2013).

${ }^{23}$ Z. Lin, C. Mei, L. Wei, Z. Sun, S. Wu, H. Huang, S. Zhang, C. Liu, Y. Feng, H. Tian, H. Yang, J. Li, Y. Wang, G. Zhang, Y. Lu, and Y. Zhao, Sci. Rep. 5, 14133 (2015).

${ }^{24}$ J. Bardeen, L. N. Cooper, and J. R. Schrieffer, Phys. Rev. 108, 1175 (1957).

${ }^{25}$ W. L. McMillan, Phys. Rev. 167, 331 (1968).

${ }^{26}$ P. B. Allen and R. C. Dynes, Phys. Rev. B 12, 905 (1975).

${ }^{27}$ J. P. Carbotte, Rev. Mod. Phys. 62, 1027 (1990).

${ }^{28}$ S. Zhang and F. J. Li, J. Appl. Phys. 111, 031301 (2012).

${ }^{29}$ D. Stamopoulos, E. Aristomenopoulou, and A. Lagogiannis, Supercond. Sci. Technol. 27, 095008 (2014).

${ }^{30}$ D. Stamopoulos and S. J. Zhang, J. Alloys Compd. 612, 34 (2014).

${ }^{31}$ M. Zeibekis, G. Vertsioti, and D. Stamopoulos, J. Phys. D: Appl. Phys. 49, 105304 (2016).

${ }^{32}$ D. Stamopoulos, A. Speliotis, and D. Niarchos, Supercond. Sci. Technol. 17, 1261 (2004)

${ }^{33}$ D. J. Thompson, M. S. M. Minhaj, L. E. Wenger, and J. T. Chen, Phys. Rev. Lett. 75, 529 (1995)

${ }^{34}$ P. Kostić, B. Veal, A. P. Paulikas, U. Welp, V. R. Todt, C. Gu, U. Geiser, J. M. Williams, K. D. Carlson, and R. A. Klemm, Phys. Rev. B 53, 791 (1996).

${ }^{35}$ M. A. López de la Torre, V. Peña, Z. Sefrioui, D. Arias, C. Leon, J. Santamaria, and J. L. Martinez, Phys. Rev. B 73, 052503 (2006).

${ }^{36}$ M. J. R. Sandim, D. Stamopoulos, L. Ghivelder, S. C. V. Lim, and A. D. Rollett, J. Supercond. Nov. Magn. 23, 1533 (2010).

${ }^{37}$ S. Y. Savrasov, D. Y. Savrasov, and O. K. Andersen, Phys. Rev. Lett. 72, 372 (1994).

${ }^{38}$ C. M. Perlov and C. Y. Fong, Phys. Rev. B 29, 1243 (1984).

${ }^{39}$ B. N. Harmon and S. K. Sinha, Phys. Rev. B 16, 3919 (1977).

${ }^{40}$ G. Q. Huang, New J. Phys. 13, 093023 (2011).

${ }^{41}$ X. L. Zhang and W. M. Liu, Sci. Rep. 5, 8964 (2015).

${ }^{42}$ M. Suda, Y. Kawasugi, T. Minari, K. Tsukagoshi, R. Kato, and H. M. Yamamoto, Adv. Mater. 26, 3490 (2014). 